\title{
Hybrid Tribo-Piezo-Electric Nanogenerator with Unprecedented Performance Based on Ferroelectric Composite Contacting Layers
}

\author{
Linards Lapčinskis, ${ }^{\dagger}$ (i) Kaspars Mălnieks, ${ }^{\dagger}$ Artis Linarts, ${ }^{\dagger}$ Juris Blūms, $^{\dagger}$ Krišjānis Šmits, ${ }^{\S}$ \\ Martin Järvekülg," Maris Knite, ${ }^{\dagger}$ and Andris Sutka*,‡0 \\ ${ }^{\dagger}$ Institute of Technical Physics, Faculty of Materials Science and Applied Chemistry, Riga Technical University, Paula Valdena 3/7,
Riga LV1048, Latvia
${ }^{\ddagger}$ Research Laboratory of Functional Materials Technology, Faculty of Materials Science and Applied Chemistry, Riga Technical
University, Paula Valdena 3/7, Riga LV1048, Latvia
${ }^{\S}$ Laboratory of Solid State Radiation Physics, Institute Solid State Physics, University of Latvia, Kengaraga 8, Riga LV1063, Latvia
${ }^{\|}$Institute of Physics, University of Tartu, W. Ostwaldi 1, Tartu 50411, Estonia
}

Supporting Information

ABSTRACT: It was recently reported that more efficient triboelectric nanogenerator (TENG)-like devices can be prepared using inversely polarized ferroelectric films made of same material as the contacting layers. In the present work, a clear correlation between the piezoelectric response of inversely polarized ferroelectric PVDF/ $\mathrm{BaTiO}_{3}$ nanocomposite films and the performance of the TENG-like device based on these films is demonstrated. This observation is explained by magnified electrostatic induction that is driven by piezoelectric charges and ferroelectric properties of these films. A double capacitor model is proposed that effectively portrays the interactions between ferroelectric layers during contact-separation and subsequent charge redistributions in the external circuit. The
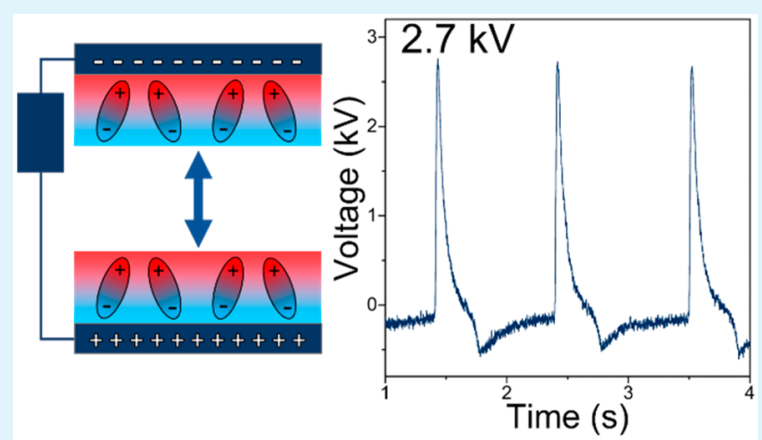
new understanding has allowed the result of 3 -fold higher open circuit voltages $\left(2.7 \mathrm{kV}\right.$ from $\left.5 \mathrm{~cm}^{2}\right)$ as compared to that of a state of the art TENG. Furthermore, findings uncover the potential for vast improvement in the field of nanogenerators for mechanical energy harvesting as a significantly better piezoelectric performance of flexible nanogenerators has been reported elsewhere.

KEYWORDS: nanogenerators, triboelectricity, piezoelectricity, ferroelectricity, poly(vinylidene fluoride)

$\mathrm{T}$ riboelectric nanogenerators (TENGs) are intriguing mechanical energy-harvesting devices that could power small portable devices ${ }^{1}$ and detectors ${ }^{2}$ or charge batteries. ${ }^{3}$ TENGs can also act as self-powered sensor elements for mechanical displacement, chemical or acoustic sensors, and biomedical monitors. The working principles of TENG are based on friction-related contact electrification. Most commonly, a TENG consists of two connected conductive electrode layers from which at least one is covered with a polymer insulator. ${ }^{4}$ These two surfaces from distinct materials are then contacted and separated, creating opposite sign net charges on the surfaces, which in turn induce charges on underlying conductive electrodes. During separation the electric potential difference is established, and electrons are driven to flow between the two electrodes in order to balance the electric potential difference. ${ }^{4}$ For surface charge formation a variety of mechanisms have been proposed. One of the widely accepted mechanisms is electron transfer between contacted materials. ${ }^{4,5}$ However, this theory has some flaws; e.g., polymer materials generally do not contain free electrons, and therefore, other mechanisms have been put forward. Mass or ion transfer from the polymer in this context seems more credible than electron transfer. ${ }^{6-10}$ This working mechanism involves formation of positively and negatively charged mosaictype fragments on each surface that result in net surface charge., 90

Recently, several research groups have demonstrated that the performance of TENG can be enhanced using ferroelectric films as contacting surfaces. ${ }^{11-18}$ Polarized ferroelectric layers have been considered to have a more appropriate work function for electron transfer between polymer layers. ${ }^{11,17}$ However, recently it has been proposed that the enhancement actually comes from piezoelectric charges, and performance can be significantly improved by contacting inversely polarized ferroelectric films from the same material due to magnified electrostatic induction. ${ }^{18}$ It appears that the piezoelectric charges created on the ferroelectric layers during contacting (pressing) drive electrostatic induction and enhance the overall 
performance of TENG. ${ }^{18}$ It is also noteworthy that in case of inversely polarized ferroelectric contacting surfaces there is no need to combine distinct materials according to their position in triboelectric series, because even moderate piezoelectric charges can be sufficient for high performance. ${ }^{18}$

However, the complete working mechanism of TENG based on inversely polarized ferroelectric layers is not clear. Piezoelectric charges which form during contacting two ferroelectric films in a TENG device are too small to observe high current and voltage upon separation. The same situation includes a surface charge forming by contacting-separating two films from the identical material and electrostatic induction from an electric field arising from permanent dipoles. At the same time, in the present work we demonstrate an obvious correlation between the magnitude of piezoelectric charges and the performance of TENG. We are building our device from a well-established $\mathrm{PVDF} / \mathrm{BaTiO}_{3}$ nanocomposite that has been reported for usage in both piezoelectric nanogenerators (PENGs) and TENGs. PVDF is a wellknown ferroelectric polymer in which the piezoelectric charge density can be increased by adding $\mathrm{BaTiO}_{3}$ nanoparticles. ${ }^{11-18}$ In the TENG setup, ferroelectric $\mathrm{PVDF} / \mathrm{BaTiO}_{3}$ nanocomposites have been contacted with other materials according to established knowledge of the triboelectric effect, but here we use identical two $\mathrm{PVDF} / \mathrm{BaTiO}{ }_{3}$ contacting layers that are inversely polarized. As a result, we present a 3 -fold higher output voltage than that from a state of the art TENG device. ${ }^{19}$ We show that the observed high performance can be explained by introducing a double capacitor model where capacitance of the device changes twice upon contacting and separating, thus varying potential and causing current flow between two conductive plates in the TENG device.

Ferroelectric contacting electrodes for a TENG device were prepared from a PVDF solution in dimethylformamide (DMF) by spin-coating in combination with immersion-precipitation schematically demonstrated in Figure $1 \mathrm{a} . \mathrm{BaTiO}_{3}$ nanoparticles in a wide compositional range $(0-35$ vol \%) were ultrasonically dispersed in PVDF solution and spin-coated on an indium-tin oxide (ITO) substrate. In the next step, the freshly spin-coated films were immersed in antisolvent (methanol), thus triggering the polymer precipitation and formation of porous structures. The porous PVDF structures formed in less than $1 \mathrm{~min}$. All prepared sample compositions presented porous nanostructures as seen in the scanning electron microscopy (SEM) image in Figure 1b. The precipitation method is well-adopted by industry to manufacture nanoporous polymer membranes ${ }^{20}$ and should easily facilitate largescale production of highly porous contacting layers for TENGlike devices. This approach is apparently highly competitive with the other methods such as templating or molding, ${ }^{19,21,22}$ electrospinning, ${ }^{23}$ and reactive ion or chemical etching, ${ }^{24,25}$ methods that have been applied for preparation of high specific surface area polymeric layers for TENG devices. A more detailed description of the preparation of samples, characterization, and measurement methods is presented in Supporting Information in the experimental methods details.

The homogeneity of the distribution of $\mathrm{BaTiO}_{3}$ nanoparticles into the polymer matrix was studied by transmission electron microscopy (TEM) and energy-dispersive X-ray spectroscopy (EDX) analysis. As can be seen from images in Figure $1 \mathrm{c}, \mathrm{d}, \mathrm{BaTiO}_{3}$ nanoparticles are well-dispersed throughout the PVDF matrix. It is important to achieve a higher dispersion quality, because a higher homogeneity yields a
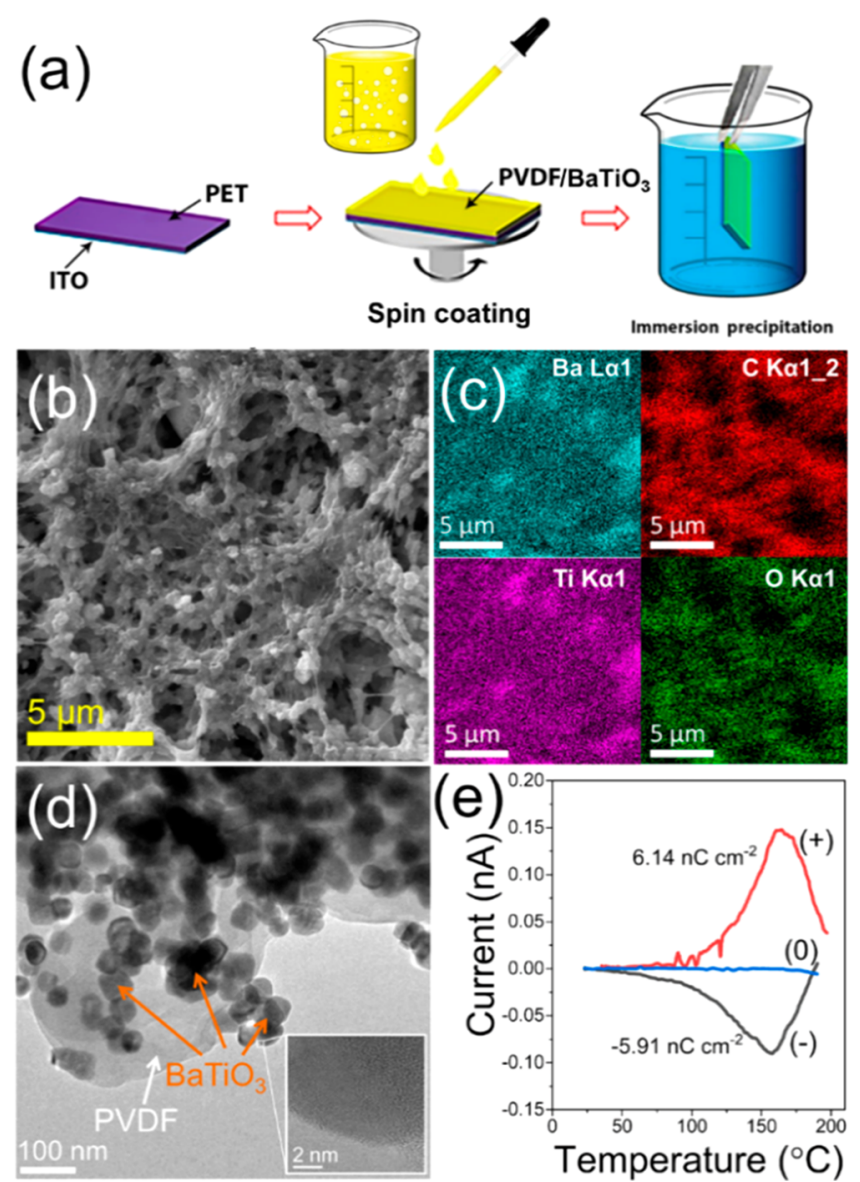

Figure 1. Schematic representation of sample film preparation by immersion-precipitation (a). Image b shows an SEM micrograph for a $\mathrm{BaTiO}_{3} / \mathrm{PVDF}$ nanocomposite with $\mathrm{BaTiO}_{3}$ at $25 \mathrm{vol} \%$ content. The same sample was analyzed by EDX (c) and TEM (d) to demonstrate homogeneous $\mathrm{BaTiO}_{3}$ nanoparticle dispersion throughout the PVDF matrix. Graph e demonstrates depolarization current curves for inversely polarized (red and gray) and nonpolarized (blue) layers of the same material showing the charge stored by ferroelectric material.

higher piezoelectric response. ${ }^{26}$ Particles in agglomerates are not free to move, limiting the change in dipole moments. ${ }^{26}$

Samples were prepared from both $\mathrm{BaTiO}_{3} / \mathrm{PVDF}$ and neat PVDF, and identical electrical measurements were conducted on poled as well as nonpoled samples. The occurrence of ferroelectric phases of PVDF before and after poling was confirmed by attenuated total reflectance Fourier transform infrared spectroscopy (ATR-FTIR) measurements, which also revealed low crystallinity of as-prepared nonpolarized PVDF (see Supporting Information Figure S1). After heating or poling (heating in electric field) at $100{ }^{\circ} \mathrm{C}$, the $\alpha, \beta$, and $\gamma$ phases were dominantly presented in the sample films (Supporting Information Figures S2 and S3).

Thermally stimulated depolarization current (TSDC) measurements of polarized $\mathrm{PVDF} / \mathrm{BaTiO}_{3}$ show that in the temperature range $130-180{ }^{\circ} \mathrm{C}$ ferroelectric composites undergo dipole relaxation within the crystalline phase (Figure $1 \mathrm{e})$. For positively $(+)$ and negatively $(-)$ polarized layers, induced charge density is, respectively, 6.14 and $5.91 \mathrm{nC} \mathrm{cm}^{-2}$. For nonpolarized $\mathrm{PVDF} / \mathrm{BaTiO}_{3}(0)$, the TSDC technique did not show charge release during heating. 
Piezo-regime
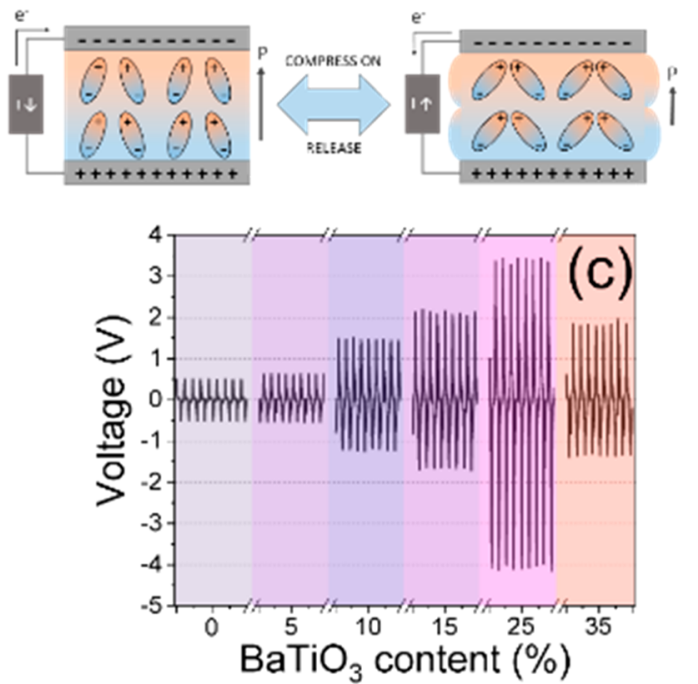

(a)

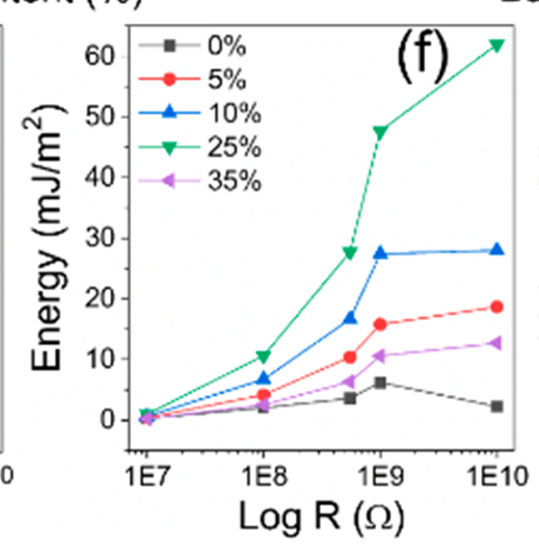

Tribo-regime
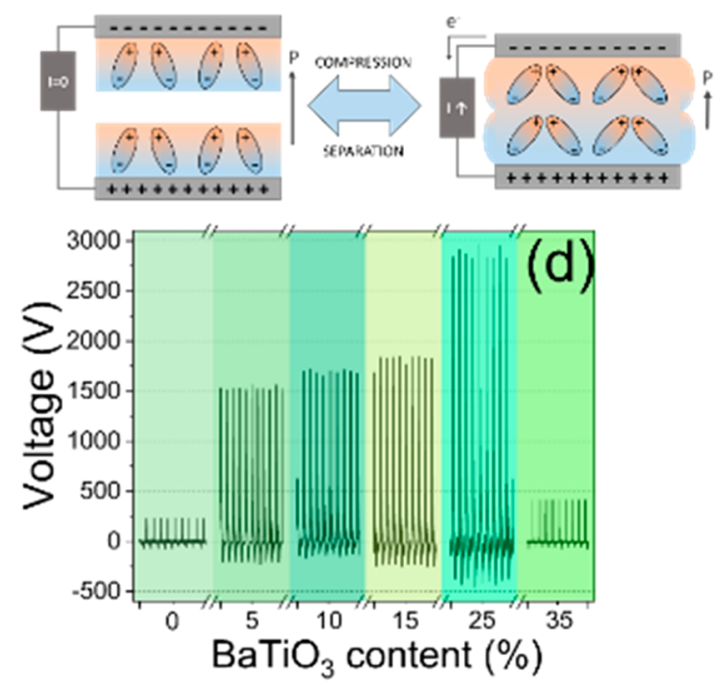
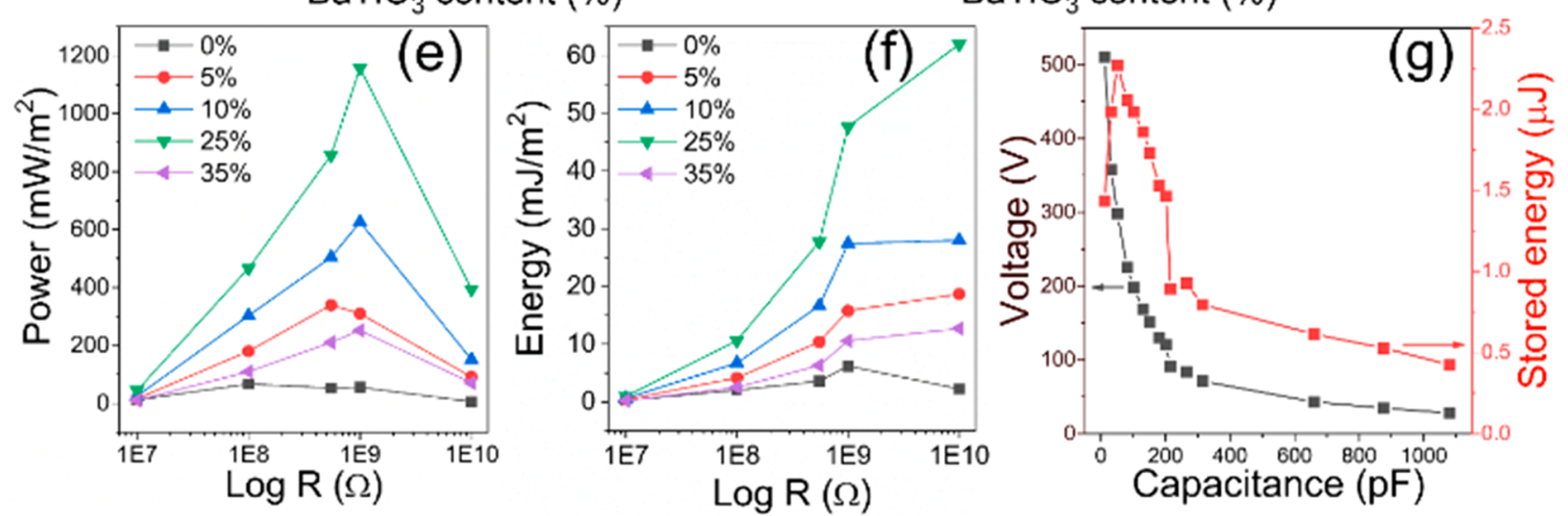

Figure 2. Schematic representation of piezo (a) and TENG (b) testing regimes. The open circuit output voltage measured at load resistance $1 \times$ $10^{10} \Omega$ in piezo (c) and TENG (d) regimes. Graphs at the bottom show power (e) and energy (f) for different compositions tested in the TENG regime. Energy stored in the capacitor $(\mathrm{g})$ was determined for the nanocomposite showing the highest performance $\left(\mathrm{PVDF} / \mathrm{BaTiO}_{3} 25 \mathrm{vol} \%\right)$.

Immersion-precipitation derived poled PVDF and their composites were tested in compression mode (Figure 2a) to assess their piezoelectric response. As expected, the reference samples that were not poled generated no detectable voltage. Compositions with $\mathrm{BaTiO}_{3}$ exhibited higher open circuit voltage $\left(V_{\mathrm{OC}}\right)$ than bare PVDF when force was applied. The higher piezoelectric response of $\mathrm{BaTiO}_{3} / \mathrm{PVDF}$ nanocomposites in comparison to bare PVDF is well-described in the literature and explained by an increased number of piezoelectric dipoles in the film. ${ }^{27-29}$ For bare polarized PVDF, a piezoelectric $V_{\mathrm{OC}}$ value of $0.5 \mathrm{~V}$ was measured. $V_{\mathrm{OC}}$ was found to gradually increase with $\mathrm{BaTiO}_{3}$ content until saturation, followed by a decline in voltages at higher concentrations (Figure 2c). The latter can be attributed to degrading electromechanical coupling effects (i.e., deformability). ${ }^{26} 25$ vol \% was found to be the optimal concentration of $\mathrm{BaTiO}_{3}$ in PVDF, resulting in the highest $V_{\mathrm{OC}}$ of $4.0 \mathrm{~V}$ in the negative direction (Figure 2c).

Prepared inversely polarized ferroelectric films were subsequently used as contacting layers in a TENG device (Figure 2b). Identical nanocomposite films were deposited on the contacting surfaces, followed by poling to establish inverse polarization. As can be seen from Figure $2 d$, just like in piezoelectric measurements, compositions containing $\mathrm{BaTiO}_{3}$, until certain loading, show a higher open circuit voltage in the
TENG regime, clearly indicating a correlation between piezoelectric response and the corresponding performance of a nanocomposite film TENG device. For example, the generator where inversely polarized bare PVDF was used as contacting layers produced a $V_{\mathrm{OC}}$ value of $250 \mathrm{~V}$, while the generator from the $\mathrm{BaTiO}_{3} / \mathrm{PVDF}$ nanocomposite with a nanoparticle content of $25 \mathrm{vol} \%$ generated a $V_{\mathrm{OC}}$ value of $2700 \mathrm{~V}$ from $5 \mathrm{~cm}^{2}$. Boosted performance of nanocomposite TENG devices relates to a higher ferroelectric polarization of layers, which is evident not only in piezoelectricity but also as an increased charge density transferred between two electrodes in a TENG device. As $\mathrm{BaTiO}_{3}$ content increases to the optimal content, the net polarization of the ferroelectric layer grows. The instant power density, calculated by Joule's law ( $P=$ $V^{2} R^{-1}$ ), reached $1.157 \mathrm{~W} \mathrm{~m}^{-2}$ for the TENG device with the highest $V_{\mathrm{OC}}$ under an optimized load resistance of $1 \times 10^{9} \Omega$ (Figure 2e), whereas the integral $E=\int P \mathrm{~d} t$ yielded $65.95 \mathrm{~mJ}$ $\mathrm{m}^{-2}$. The dependence of the calculated power density and energy density on load resistance for different compositions under the TENG regime is depicted in Figure 2e,f, respectively. The results of the corresponding voltage measurements for different nanocomposites in the TENG regime under different loads are demonstrated in Supporting Information Figures S4S9. 
The composition exhibiting the best performance (25 vol \% $\mathrm{BaTiO}_{3}$ ) was used to charge a variable capacitor circuit. Figure $2 \mathrm{~g}$ depicts energy stored in the capacitor (and the corresponding voltage of the charged state) for each capacitor value after contact between two ferroelectric layers with $5 \mathrm{~cm}^{2}$ contact area. The highest energy stored in a capacitor after a single contact-separation step $(2.27 \mu \mathrm{J}$ calculated by $E=$ $\left.0.5 C V^{2}\right)$ was reached when the capacitance of the capacitor circuit was set to approximately $50 \mathrm{pF}$.

Due to their intrinsic properties, the piezoelectric performances of the ferroelectric materials applied here are rather poor compared to state of art piezoelectric nanogenerators. ${ }^{26,29,30}$ The achieved TENG performance of the device based on the same inversely polarized ferroelectric films, however, matches the best reported values for TENGs. We also provide proof of clear correlation between the performances of the same material in these two regimes. Therefore, as state of the art PENG devices presented in the literature generate up to $V_{\mathrm{OC}}=$ $209 \mathrm{~V},{ }^{30,31}$ significant improvement in the performance of TENG-like devices should be possible if the corresponding materials are used as inversely polarized films in the TENG regime. However, this prediction assumes that the voltage and current observed in these PENG devices have been correctly attributed to piezoelectric charges and are not exaggerated due to unaccounted friction.

However, the question arises regarding how a large voltage can be generated by a piezoelectric charge, because when we compare charges (calculated as $q=\int i_{\mathrm{SC}} \mathrm{d} t$ ) generated by the $\mathrm{PVDF} / \mathrm{BaTiO}_{3}$ composite based on a TENG device with optimal $\mathrm{BaTiO}_{3}$ content (25 vol \%) in the piezoelectric and TENG regimes in a wide applied pressure range, we see that the piezoelectric charge is 2 orders of magnitude smaller than the charge observed in the TENG regime (measured charge values in different regimes for PVDF 25 vol $\% \mathrm{BaTiO}_{3}$ are presented in Tables $\mathrm{S} 1-\mathrm{S} 3$ ). The increase in charge upon separation cannot be related to electrostatic effects, because charges $\left(2.83 \mathrm{nC} \mathrm{cm}^{-2}\right)$ observed between oscillating electrodes in the noncontact mode (ferroelectric layers are moved toward each other similarly as in TENG measurements, but without physical contact) only partially explain the high TENG values.

The piezoelectric response allows calculation of the piezoelectric coefficient effective value $d_{33}$ which for the $25 \%$ $\mathrm{BaTiO}_{3}$ composite reaches $47.9 \mathrm{pC} \mathrm{N}^{-1}$ (calculation shown in Figure S10 and eq S1) while values reported in the literature for similar ferroelectric $\mathrm{PVDF} / \mathrm{BaTiO}_{3}$ composites range from 2.7 to $25 \mathrm{pC} \mathrm{N}^{-1} \cdot{ }^{32,33}$ A greater piezoelectric coefficient can be related to a significantly larger deformability due to high porosity (with consideration of bulk density, weight, and thickness, the porosity was estimated to be around 70\%) of the samples. Porosity provides space for local deformations in the material that are important for piezoelectric charge generation and boosts the formation of $\beta$-PVDF. ${ }^{34}$ The piezoelectric coefficient of a dense PVDF $25 \% \mathrm{BaTiO}_{3}$ composite was determined to be $9.11 \mathrm{pC} \mathrm{N}^{-1}$ which is 5 times smaller than that for the porous material used in TENG devices (Figure S10 and eq S2).

For the explanation of the potential difference change between electrodes, a double capacitor model is proposed in Figure 3. Each ferroelectric layer can be considered as an individual capacitor with surface charge density $\sigma$ induced during polarization. The potential difference between opposite surfaces of each layer can be described as

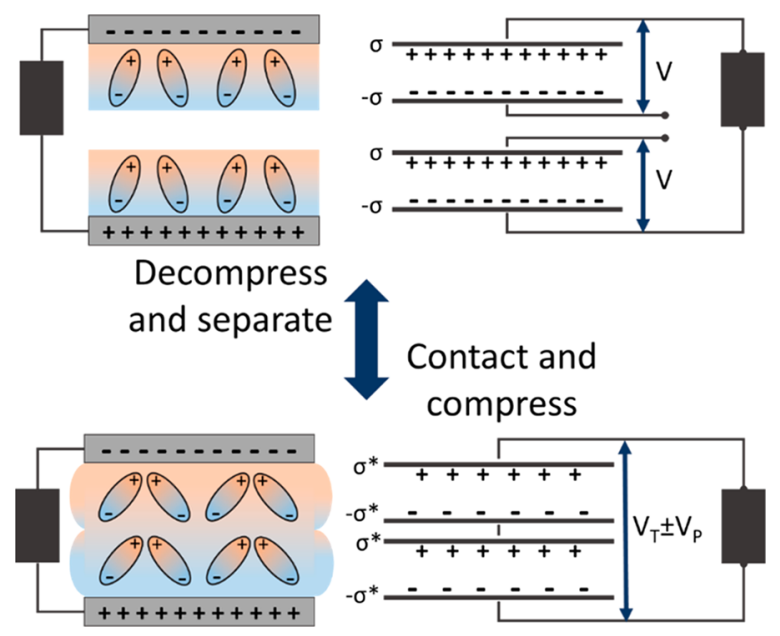

Figure 3. Double capacitor model and corresponding contactseparation stages. Dipoles in the schematic here represent both PVDF and $\mathrm{BaTiO}_{3}$ dipoles in ferroelectric layers. At first, ferroelectric layers contact and form a connected capacitor, and then layers are compressed which results in a negative piezoelectric response. Next, at a much faster rate the compressed deformation is released resulting in a positive piezoelectric response which is immediately followed by separation of both surfaces, thus disconnecting the capacitors that had formed the connected circuit.

$$
V=\frac{q}{C}=\frac{q d}{\varepsilon_{0} \varepsilon A}=\frac{\sigma d}{\varepsilon_{0} \varepsilon}
$$

By considering two inversely polarized layers as two capacitors we can conclude that the total capacitance (and correspondingly potential difference) depends on the air gap between them. As layers come together and contact, they form a circuit of two identical capacitors in series, and the total capacitance of two layers is decreasing; however, the voltage is increasing twice in accordance with eq 2 :

$$
C_{\mathrm{TOT}}=\frac{q}{V_{\mathrm{TOT}}}=\frac{C}{2}=\frac{q}{2 V}
$$

Next, compression induces piezocharge formation as dipoles deform and the net polarization of layers drops. As a result, the surface charge density decreases to $\sigma^{*}$. This is followed by fast separation of compressed ferroelectric layers where net polarization quickly rises and charge density returns to $\sigma$. When ferroelectric layers are separated, the potential difference decreases in accordance with eq 2; this in turn induces additional redistribution of induced charges between electrodes in the external circuit. The charge redistributed between electrodes is registered as current peak $\int i_{\mathrm{SC}} \mathrm{d} t=0.5\left(q_{1}+q_{2}\right)$, where $q_{1}$ and $q_{2}$ are charge densities on polarized layer surfaces, and this allows a comparison of experimental data with the model. TENG measurement data of the PVDF 25\% $\mathrm{BaTiO}_{3}$ composite were used where positively and negatively polarized layers are contacted with each other and with the nonpolarized layer.

Induced charge density in the external circuit $\left(\int i_{\mathrm{SC}} \mathrm{d} t\right)$ for positively and negatively polarized layers was determined by contacting them with the nonpolarized layer in the TENG mode. For positively and negatively polarized layers, $\int i_{\mathrm{SC}} \mathrm{d} t$ was determined to be 4.19 and $2.41 \mathrm{nC} \mathrm{cm}^{-2}$ while contact between two nonpolarized layers resulted in only $0.03 \mathrm{nC}$ $\mathrm{cm}^{-2}$, so we can assume that there is no surface charge on the nonpolarized layer. Therefore, $\int i_{\mathrm{SC}} \mathrm{d} t=0.5\left(q_{1}+q_{2}\right)$ gives us 
that charge density $q_{1}$ on the surface of the positively polarized layer is $8.38 \mathrm{nC} \mathrm{cm}^{-2}$ while $q_{2}$ on the negatively polarized layer surface is $4.82 \mathrm{nC} \mathrm{cm}$.

When two capacitors with equal capacitances but with different charge density states are connected in series then the charge density on connected surfaces is $q_{1}-q_{2}$, but on outer surfaces it is $0.5\left(q_{1}-q_{2}\right)$ and $0.5\left(q_{2}-q_{1}\right)$. In our case we obtain that charge density on each of the inner surfaces of two connected capacitors is $3.56 \mathrm{nC} \mathrm{cm}^{-2}$ while it is $1.78 \mathrm{nC} \mathrm{cm}-2$ on outer surfaces. This means that redistributed charge density in accordance with the double capacitor model presented in Figure 3 is $8.38-1.78=-(4.82-1.78)=6.60 \mathrm{nC} \mathrm{cm}^{-2}$. The induced charge density in the TENG measurement $(6.55 \mathrm{nC}$ $\mathrm{cm}^{-2}$ ) when negatively and positively polarized layers are contacted is very similar to one obtained from model calculations.

In conclusion, highly porous piezoelectric nanocomposites based on $\mathrm{BaTiO}_{3} /$ PVDF were successfully prepared by immersion-precipitation. It was observed that the piezoelectric response rises with increasing $\mathrm{BaTiO}_{3}$ loading until 25 vol \% content is reached. Sample films were further used in TENG devices as contacting layers on electrodes, and a clear correlation was observed between piezoelectric response and TENG performance. The PVDF/ $\mathrm{BaTiO}_{3}$ nanocomposite with optimal composition shows $47.9 \mathrm{pC} \mathrm{N}^{-1}$ as the piezoelectric coefficient, higher than that usually reported for such composites, and in TENG mode a record high $2.7 \mathrm{kV} V_{\mathrm{OC}}$ value is achieved. However, our results also indicate that piezoelectric charges cannot be solely responsible for great TENG performance; thus, a "double capacitor model" has been introduced. The mechanism involves interaction between two charged ferroelectric layers during contact-separation, and contacted inversely polarized layers can be considered as capacitors connected in series. Air gap formation during separation rapidly decreases the total capacitance while the potential difference increases. The induced charge redistribution in the external circuit is registered as a current. Predictions of our model also hold true when experimentally obtained charge density $\left(6.55 \mathrm{nC} \mathrm{cm}^{-2}\right)$ values are compared with ones obtained from model calculations $\left(6.60 \mathrm{nC} \mathrm{cm}^{-2}\right)$. As triboelectric research continues to thrive and expand, we anticipate that our work will help the TENG community to further improve the performance of ferroelectric-based triboelectric devices.

\section{AUTHOR INFORMATION}

\section{Corresponding Author}

*E-mail: Andris.Sutka@rtu.lv.

\section{ORCID}

Linards Lapčinskis: 0000-0002-5048-2429

Andris Sutka: 0000-0002-5739-0164

\section{Notes}

The authors declare no competing financial interest.

\section{ACKNOWLEDGMENTS}

This research was supported by the European Regional Development Fund within the project "Hybrid energy harvesting systems" 1.1.1.1./16/A/013.

\section{REFERENCES}

(1) Kim, W. G.; Kim, D.; Jeon, S. B.; Park, S. J.; Tcho, I. W.; Jin, I. K.; Han, J. K.; Choi, Y. K. Multidirection and Multiamplitude Triboelectric Nanogenerator Composed of Porous Conductive Polymer with Prolonged Time of Current Generation. Adv. Energy Mater. 2018, 8, 1800654.

(2) Wen, Z.; Chen, J.; Yeh, M.; Guo, H.; Li, Z.; Fan, X.; Zhang, T.; Zhu, L.; Wang, Z. L. Blow-driven triboelectric nanogenerator as an active alcohol breath analyzer. Nano Energy 2015, 16, 38-46.

(3) Pu, X.; Li, L.; Song, H.; Du, C.; Zhao, Z.; Jiang, C.; Cao, G.; Hu, W.; Wang, Z. L. A Self-Charging Power Unit by Integration of a Textile Triboelectric Nanogenerator and a Flexible Lithium-Ion Battery for Wearable Electronics. Adv. Mater. 2015, 27, 2472-2478.

(4) Wang, Z. L. Triboelectric Nanogenerators as New Energy Technology for Self-Powered Systems and as Active Mechanical and Chemical Sensors. ACS Nano 2013, 7, 9533-9557.

(5) Lin, S.; Xu, L.; Xu, C.; Chen, X.; Wang, A. C.; Zhang, B.; Lin, P.; Yang, Y.; Zhao, H.; Wang, Z. L. Electron Transfer in Nanoscale Contact Electrification: Effect of Temperature in the MetalDielectric Case. Adv. Mater. 2019, 31, 1808197.

(6) Horn, R. G.; Smith, D. T. Contact Electrification and Adhesion Between Dissimilar Materials. Science 1992, 256, 362-364.

(7) Baytekin, H. T.; Baytekin, B.; Incorvati, J. T.; Grzybowski, B. A. Material Transfer and Polarity Reversal in Contact Charging. Angew. Chem., Int. Ed. 2012, 51, 4843-4847.

(8) Baytekin, H. T.; Baytekin, B.; Hermans, T. M.; Kowalczyk, B.; Grzybowski, B. A. Control of Surface Charges by Radicals as a Principle of Antistatic Polymers Protecting Electronic Circuitry. Science 2013, 341, 1368-1371.

(9) Baytekin, H. T.; Patashinski, A. Z.; Branicki, M.; Baytekin, B.; Soh, S.; Grzybowski, B. A. The Mosaic of Surface Charge in Contact Electrification. Science 2011, 333, 308-312.

(10) Apodaca, M.; Wesson, P.; Bishop, K.; Ratner, M.; Grzybowski, B. Contact Electrification between Identical Materials. Angew. Chem., Int. Ed. 2010, 49, 946-949.

(11) Bai, P.; Zhu, G.; Zhou, Y. S.; Wang, S.; Ma, J.; Zhang, G.; Wang, Z. L. Dipole-moment-induced effect on contact electrification for triboelectric nanogenerators. Nano Res. 2014, 7 (7), 990-997.

(12) Seung, W.; Yoon, H.-J.; Kim, T. Y.; Ryu, H.; Kim, J.; Lee, J.-H.; Lee, J. H.; Kim, S.; Park, Y. K.; Park, Y. J.; Kim, S.-W. Boosting Power-Generating Performance of Triboelectric Nanogenerators via Artificial Control of Ferroelectric Polarization and Dielectric Properties. Adv. Energy Mater. 2017, 7, 1600988.

(13) Suo, G.; Yu, Y.; Zhang, Z.; Wang, S.; Zhao, P.; Li, J.; Wang, X. Piezoelectric and Triboelectric Dual Effects in Mechanical-Energy Harvesting Using $\mathrm{BaTiO}_{3} /$ Polydimethylsiloxane Composite Film. ACS Appl. Mater. Interfaces 2016, 8, 34335-34341.

(14) Chun, J.; Kim, J. W.; Jung, W. S.; Kang, C. Y.; Kim, S. W.; Wang, Z. L.; Baik, J. M. Mesoporous pores impregnated with Au nanoparticles as effective dielectrics for enhancing triboelectric nanogenerator performance in harsh environments. Energy Environ. Sci. 2015, 8, 3006-3012.

(15) Yang, X.; Daoud, W. A. Synergetic effects in composite-based flexible hybrid mechanical energy harvesting generator. J. Mater. Chem. A 2017, 5, 9113-9121.

(16) Choi, Y. S.; Jing, Q.; Datta, A.; Boughey, C.; Kar-Narayan, S. A triboelectric generator based on self-poled Nylon-11 nanowires fabricated by gas-flow assisted template wetting. Energy Environ. Sci. 2017, 10, 2180-2189. 
(17) Lee, K. Y.; Kim, S. K.; Lee, J.-H.; Seol, D.; Gupta, M. K.; Kim, Y.; Kim, S.-W. Controllable Charge Transfer by Ferroelectric Polarization Mediated Triboelectricity. Adv. Funct. Mater. 2016, 26, 3067-3073.

(18) Sutka, A.; Mālnieks, K.; Linarts, A.; Timusk, M.; Jurķāns, V.; Gorņevs, I.; Blūms, J.; Bērziņa, A.; Joost, U.; Knite, M. Inversely polarised ferroelectric polymer contact electrodes for triboelectric-like generators from identical materials. Energy Environ. Sci. 2018, 11, 1437-1443.

(19) Chun, J.; Ye, B. U.; Lee, J. W.; Choi, D.; Kang, C. Y.; Kim, S. W.; Wang, Z. L.; Baik, J. M. Boosted output performance of triboelectric nanogenerator via electric double layer effect. Nat. Commun. 2016, 7, 12985.

(20) van de Witte, P.; Dijkstra, P. J.; van den Berg, J. W. A.; Feijen, J. Phase separation processes in polymer solutions in relation to membrane formation. J. Membr. Sci. 1996, 117, 1-31.

(21) Fan, F. R.; Lin, L.; Zhu, G.; Wu, W.; Zhang, R.; Wang, Z. L. Transparent Triboelectric Nanogenerators and Self-Powered Pressure Sensors Based on Micropatterned Plastic Films. Nano Lett. 2012, 12, 3109-3114.

(22) Han, M.; Zhang, X. S.; Meng, B.; Liu, W.; Tang, W.; Sun, X.; Wang, W.; Zhang, H. r-Shaped Hybrid Nanogenerator with Enhanced Piezoelectricity. ACS Nano 2013, 7, 8554-8560.

(23) Zheng, Y.; Cheng, L.; Yuan, M.; Wang, Z.; Zhang, L.; Qin, Y.; Jing, T. An electrospun nanowire-based triboelectric nanogenerator and its application in a fully self-powered UV detector. Nanoscale 2014, 6, 7842-7846.

(24) Fan, X.; Chen, J.; Yang, J.; Bai, P.; Li, Z.; Wang, Z. L. Ultrathin, Rollable, Paper-Based Triboelectric Nanogenerator for Acoustic Energy Harvesting and Self-Powered Sound Recording. ACS Nano 2015, 9, 4236-4243.

(25) Lin, Z. H.; Cheng, G.; Li, X.; Yang, P. K.; Wen, X.; Wang, Z. L. A multi-layered interdigitative-electrodes-based triboelectric nanogenerator for harvesting hydropower. Nano Energy 2015, 15, 256265.

(26) Lee, E. J.; Kim, T. Y.; Kim, S.-W.; Jeong, S.; Choi, Y.; Lee, S. Y. High-performance piezoelectric nanogenerators based on chemicallyreinforced composites. Energy Environ. Sci. 2018, 11, 1425-1430.

(27) Zhao, Y.; Liao, Q.; Zhang, G.; Zhang, Z.; Liang, Q.; Liao, X.; Zhang, Y. High output piezoelectric nanocomposite generators composed of oriented $\mathrm{BaTiO}_{3} \mathrm{NPs@PVDF}$. Nano Energy 2015, 11, 719-727.

(28) Siddiqui, S.; Kim, D.-I.; Duy, L. T.; Nguyen, M. T.; Muhammad, S.; Yoon, W.-S.; Lee, N.-E. High-performance flexible lead-free nanocomposite piezoelectric nanogenerator for biomechanical energy harvesting and storage. Nano Energy 2015, 15, 177-185.

(29) Shin, S.-H.; Kim, Y.-H.; Lee, M. H.; Jung, J.-Y.; Nah, J. Hemispherically Aggregated $\mathrm{BaTiO}_{3}$ Nanoparticle Composite Thin Film for High-Performance Flexible Piezoelectric Nanogenerator. ACS Nano 2014, 8, 2766-2773.

(30) Gu, L.; Cui, N.; Cheng, L.; Xu, Q.; Bai, S.; Yuan, M.; Wu, W.; Liu, J.; Zhao, Y.; Ma, F.; Qin, Y.; Wang, Z. L. Flexible Fiber Nanogenerator with 209 V Output Voltage Directly Powers a LightEmitting Diode. Nano Lett. 2013, 13, 91-94.

(31) Yi, F.; Lin, L.; Niu, S.; Yang, P. K.; Wang, Z.; Chen, J.; Zhou, Y.; Zi, Y.; Wang, J.; Liao, Q.; Zhang, Y.; Wang, Z. L. StretchableRubber-Based Triboelectric Nanogenerator and Its Application as Self-Powered Body Motion Sensors. Adv. Funct. Mater. 2015, 25, 3688-3696.

(32) Li, R.; Zhao, Z.; Chen, Z.; Pei. Novel BaTiO3/PVDF composites with enhanced electrical properties modified by calcined $\mathrm{BaTiO}_{3}$ ceramic powders. Mater. Express 2017, 7, 536-540.

(33) Olszowy, M. Piezoelectricity and dielectric properties of PVDF/ $/ \mathrm{BaTiO}_{3}$ composites. Proc. SPIE 1996, 3181, 69-72.

(34) Chang, C.; Tran, V. H.; Wang, J.; Fuh, Y. K.; Lin, L. DirectWrite Piezoelectric Polymeric Nanogenerator with High Energy Conversion Efficiency. Nano Lett. 2010, 10, 726-731. 